\title{
Target Tracking for Wireless Localization Systems with Degraded Measurements and Quantization Effects
}

\author{
Xingzhen Bai, Zidong Wang, Lei Zou and Cheng Cheng
}

\begin{abstract}
In this paper, the target tracking problem is investigated for a class of wireless localization systems with degraded measurements and quantization effects. The measurement degradations induced by unreliable channels are characterized by a random parameter matrix, and the quantization effects are modeled by sector-bounded uncertainties. A recursive filtering algorithm is proposed in order to track the plant states as accurately as possible. An upper bound on the filtering error covariance is first derived and such an upper bound is then minimized by properly designing the filter gain at each sampling instant. The desired filter parameters are obtained by solving two sets of Riccati-like difference equations that are in a recursive form suitable for the online application. Finally, a simulation experiment is carried out for the scenario of personnel localization/tracking problems in a mine industrial site and the effectiveness of the proposed filter method is demonstrated.
\end{abstract}

Index Terms-Target tracking; recursive filtering; wireless localization system; degraded measurement; quantization.

\section{INTRODUCTION}

In industrial manufacturing processes especially safetycritical systems, it is highly desirable to avoid adventitious accidents which might lead to unexpected injuries and losses. In this case, developing effective personnel/equipment monitoring system is of practical significance in preserving the operation and personnel safety. In particular, personnel positioning plays a key role for personnel monitoring to ensure the safety of personnel working in a hazardous workplace. In engineering practice, wireless localization systems are usually employed for safety control based on distributed sensing strategies [1], [6], [20], [23], [25], [26]. Briefly speaking, the main function of a wireless localization system is to track workers and mobile equipments in industrial sites, thereby helping to achieve the accurate perception of security information in the industrial manufacturing process.

In practical systems, many different localization/tracking methods have been introduced to meet specific industry requirements. In general, the proposed localization/tracking approaches are implemented based mainly on the distance

This work was supported in part by the Research Fund for the Taishan Scholar Project of Shandong Province of China, in part by the National Natural Science Foundation of China under Grants 61525305, 61773400, 61703245 and 61703242, in part by the Royal Society of the U.K., and in part by the Alexander von Humboldt Foundation of Germany. (Corresponding author Xingzhen Bai)

X. Bai, L. Zou and C. Cheng are with the College of Electrical Engineering and Automation, Shandong University of Science and Technology, Qingdao 266590, China (Email: xzbai@163.com; zouleicup@gmail.com; chengchenghs@126. com).

Z. Wang is with the Department of Computer Science, Brunel University London, Uxbridge, Middlesex, UB8 3PH, U.K. (Email: Zidong.Wang@brunel.ac.uk). measurement technologies which include, but are not limited to, time-of-arrival (TOA) method [43], time-difference-ofarrival (TDOA) [12] scheme, angle-of-arrival (AOA) approach [27], received signal strength (RSS) strategy [2], and their combinations [5], [24], [32]. In order to obtain the precise target position, a two-step localization scheme is usually utilized in the localization approaches [38], [43]. First, the sensors measure their distance to the target and transmit the measurement data to the corresponding leader unit (either a cluster head or a computing unit). Then, an estimate of the target position is generated based on the received measurements [33]. Note that the distance between the sensors and the target is often modeled by certain nonlinear functions and, therefore, various nonlinear filtering algorithms have been developed to deal with the target tracking problem for wireless localization systems, where the covariance matrix of the estimation error has been used to determine the next tasking node or the sampling instant so as to improve the tracking accuracy [17], [19], [39]. In [34], a new target tracking strategy has been established for wireless sensor networks, where the maximum likelihood estimation method and Kalman filtering technology have been employed to eliminate the linearizing error of the extended Kalman filtering algorithm.

Wireless communication is a typical network-based communication mode where the signal transmissions are likely affected by certain physical constraints including sensors aging and limited bandwidth [8]. Accordingly, various network-induced phenomena might occur during the wireless communication which include, for instance, missing measurements, transmission delays and degraded measurements [18], [29], [41], [42]. For the filter design issue over wireless communication, if the underlying network-induced phenomena are not properly dealt with, it would be quite difficult to achieve a satisfactory filtering performance. So far, considerable research attention has been devoted to the filtering problems with different network-induced phenomena [8], [9], [22], [28], [36], [44]. In particular, an increasing research interest has been paid to the filtering issues with measurement degradations, which serves as a frequently encountered phenomenon during the signal transmissions between the target and the sensors in wireless localization systems. Clearly, the localization performance is largely affected by the measurement accuracy and, therefore, it is practically significant to develop effective filter schemes concerning the target tracking issue on wireless localization systems with degraded measurements.

In addition to the measurement degradation, another important network-induced phenomenon in wireless communication 
is the signal quantization [47], which is caused mainly by the limited channel capacity [36]. Up to now, a series of theoretical results have been available on the filtering/estimation problems for various systems with quantization effects, see e.g. [4], [10], [15], [37], [45] and the references therein. For example, in [10], the distributed filtering problem has been studied for time-varying systems over sensor networks subject to quantization effects. In [45], the quantization technique has been adopted to decrease the transmission rates for energy saving. Note that quantization effects would lead to additional errors on the available measurements [15], which may degrade the filtering/estimation performance. Nevertheless, in the context of wireless localization systems, the corresponding results have been very few despite the importance of compensating quantization errors for guaranteeing satisfactory monitoring performance, and this constitutes another motivation for the current investigation.

In view of the above discussion, in this paper, we aim to design a recursive filtering algorithm for the target tracking issue of the wireless localization system subject to both degraded measurements and quantization effects, thereby facilitating the real-time personnel tracking and improving the safety management level in the industrial site. Considering that the signal transmission between the target and the sensors is severely affected by the unreliable wireless channel in a complicated industrial environment, a stochastic model is adopted to describe the degradation phenomenon of the distance measurement process. In addition, the signal transmission between the sensors and the filter is affected by a logarithmic quantization. A recursive filtering algorithm is designed such that an upper bound of the filtering error covariance is guaranteed and then minimized by appropriately designing the filter gain.

The main technical contributions of this paper are summarized as follows. 1) In order to reflect the reality of the constrained communication, both the degraded measurements and the signal quantization are considered in the measurement model of the wireless localization system. 2) A dedicated recursive filtering algorithm is developed to guarantee the filtering performance of the target tracking, where the desired filter gain is calculated recursively which is suitable for online application. 3) The developed filter scheme is applied to the mine industrial site to improve the performance of the personnel tracking, and is therefore propitious to enhance the safety management capability in the industrial manufacturing process.

Notation: The notation used here is fairly standard except where otherwise stated. $\mathbb{R}^{n}$ and $\mathbb{R}^{n \times m}$ denote the $n$ dimensional Euclidean space and the set of all $n \times m$ real matrices, respectively. The notation $X>Y$ (respectively, $X \geq Y$ ), where $X$ and $Y$ are real symmetric matrices, means that $X-Y$ is positive definite (respectively, positive semi-definite). $I$ denotes the identity matrix with compatible dimension. $A^{T}$ and $A^{-1}$ represent the transpose and inverse of matrix $A$, respectively. $\mathbb{E}\{x\}$ stands for the expectation of the random variable $x$. $\circ$ denotes the Hadamard product defined by $[A \circ B]_{i j}=A_{i j} \cdot B_{i j} \cdot \operatorname{tr}(\cdot)$ represents the trace of a matrix. $\operatorname{diag}\left\{X_{1}, X_{2}, \ldots, X_{n}\right\}$ stands for a block-diagonal matrix with matrices $X_{1}, X_{2}, \ldots, X_{n}$ on the diagonal. Matrices are assumed to have compatible dimensions if their dimensions are not stated explicitly.

\section{PROBLEM FORMULATION AND PRELIMINARIES}

Consider a wireless localization system deployed in the maintenance platform for the reliable operation of mine hoister as shown in Fig. 1. The target moves in the monitored area

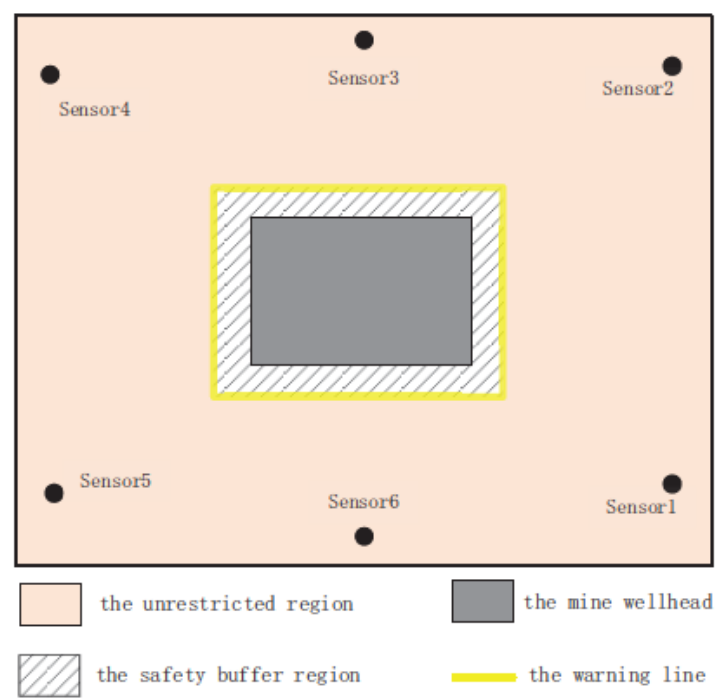

Fig. 1: Maintenance platform for the reliable operation of mine hoister

whose position and velocity are described by the following vector:

$$
x_{k}=\left[\begin{array}{llll}
x_{1}(k) & v_{1}(k) & x_{2}(k) & v_{2}(k)
\end{array}\right]^{T}
$$

where $x_{i}(k)$ and $v_{i}(k)(i=1,2)$ denote, respectively, the coordinate values of the target positions and velocities along the $x_{i}$ axis at time $t_{k}$. The dynamics of $x_{k}$ is given as follows [30], [33]:

$$
x_{k+1}=A_{k} x_{k}+B_{k} \omega_{k}
$$

where

$$
A_{k}=\left[\begin{array}{cccc}
1 & \Delta t_{k} & 0 & 0 \\
0 & 1 & 0 & 0 \\
0 & 0 & 1 & \Delta t_{k} \\
0 & 0 & 0 & 1
\end{array}\right], \quad B_{k}=\left[\begin{array}{cc}
\frac{\Delta t_{k}^{2}}{2} & 0 \\
\Delta t_{k} & 0 \\
0 & \frac{\Delta t_{k}^{2}}{2} \\
0 & \Delta t_{k}
\end{array}\right],
$$

and $\Delta t_{k}=t_{k+1}-t_{k}$ represents the time interval between two successive sampling instant of the wireless localization system. $\omega_{k} \triangleq\left[\begin{array}{ll}\omega_{1}(k) & \omega_{2}(k)\end{array}\right]^{T}$ is the white Gaussian noise with zero mean and covariance matrix $R_{\omega}$. Here, $\omega_{1}(k)$ and $\omega_{2}(k)$ are the noise components along the $x_{1}$ and $x_{2}$ axes, respectively, which are not correlated with each other.

Next, let us introduce the measurement output of system (2). Suppose that there are $m$ tasking sensors, and the corresponding measurement data of the $i$-th sensor at time $t_{k}$ is $r_{i}(k)$. The signal transmissions between the target and sensors are implemented via an unreliable wireless medium leading to measurement degradations. In this paper, $r_{i}(k)$ is described by:

$$
r_{i}(k)=\beta_{k}^{i} g_{i}\left(x_{k}\right)+\xi_{k}^{i},
$$


where the measurement coefficients $\beta_{k}^{i}(i=1, \cdots, m)$ are mutually independent random variables having the probability density function $p_{k}^{i}(s)$ on the interval $[0,1]$ with mathematical expectations $\bar{\beta}_{k}^{i}$ and variances $\nu_{k}^{i} . \xi_{k}^{i}$ is the measurement noise. $g_{i}\left(x_{k}\right)$ is the distance function given as

$$
g_{i}\left(x_{k}\right)=\sqrt{\left(x_{1}(k)-x_{1, i}^{*}\right)^{2}+\left(x_{2}(k)-x_{2, i}^{*}\right)^{2}}
$$

where $x_{1, i}^{*}$ and $x_{2, i}^{*}$ denote the coordinate values of the $i$-th sensor node along the $x_{1}$ and $x_{2}$ axes, respectively.

For notation simplicity, we let

$$
\bar{y}_{k} \triangleq\left[\begin{array}{c}
r_{1}(k) \\
r_{2}(k) \\
\vdots \\
r_{m}(k)
\end{array}\right], \quad g\left(x_{k}\right) \triangleq\left[\begin{array}{c}
g_{1}\left(x_{k}\right) \\
g_{2}\left(x_{k}\right) \\
\vdots \\
g_{m}\left(x_{k}\right)
\end{array}\right] .
$$

Then, the measured signal is rewritten as:

$$
\bar{y}_{k}=\Xi_{k} g\left(x_{k}\right)+\xi_{k}
$$

where $\xi_{k}=\left[\begin{array}{llll}\xi_{k}^{1} & \xi_{k}^{2} & \cdots & \xi_{k}^{m}\end{array}\right]^{T}$ is a zero-mean Gaussian noise sequence with covariance matrix $R_{\xi}$, and $\Xi_{k}=$ $\operatorname{diag}\left\{\beta_{k}^{1}, \beta_{k}^{2}, \cdots, \beta_{k}^{m}\right\}$ with the mathematical expectation $\bar{\Xi}=$ $\operatorname{diag}\left\{\bar{\beta}_{k}^{1}, \bar{\beta}_{k}^{2}, \cdots, \bar{\beta}_{k}^{m}\right\}$. In addition, the measured signal would be quantized before being transmitted to the filter. The quantizer $h(\cdot)=\left[\begin{array}{llll}h_{1}(\cdot) & h_{2}(\cdot) & \cdots & h_{m}(\cdot)\end{array}\right]^{T}$ is assumed to be of the logarithmic type. Particularly, for each $h_{i}(\cdot)(1 \leq i \leq m)$, the set of quantization levels is described by

$$
\begin{array}{r}
\mathcal{U}=\left\{ \pm \mu_{i}^{(j)}, \mu_{i}^{(j)}=\mathcal{X}_{j}^{i} \mu_{0}^{(j)}, i=0, \pm 1, \pm 2, \ldots\right\} \cup\{0\} \\
0<\mathcal{X}_{j}<1, \mu_{0}^{(j)}>0
\end{array}
$$

where $\mathcal{X}_{j}(j=1,2, \ldots m)$ is the so-called quantization density. The number of the quantizer level is supposed to be sufficient large. Each quantization level corresponds to a segment such that the quantizer maps the whole segment to this quantization level. The logarithmic quantizer $h_{j}(\cdot)$ is given by

$$
h_{j}\left(r_{j}(k)\right)= \begin{cases}\mu_{i}^{(j)}, & \frac{1}{1+\delta_{j}} \mu_{i}^{(j)}<\bar{y}_{k}^{(j)} \leq \frac{1}{1-\delta_{j}} \mu_{i}^{(j)} \\ 0, & \bar{y}_{k}^{(j)}=0 \\ -h_{j}\left(r_{j}(k)\right), & \bar{y}_{k}^{(j)}<0\end{cases}
$$

where $\delta_{j}=\left(1-\mathcal{X}_{j}\right) /\left(1+\mathcal{X}_{j}\right)$. It follows from (7) that $h_{j}\left(r_{j}(k)\right)$ can be expressed as $h_{j}\left(r_{j}(k)\right)=\left(1+\Delta_{k}^{(j)}\right) r_{j}(k)$ for certain $\Delta_{k}^{(j)}$ with $\left|\Delta_{k}^{(j)}\right| \leq \delta_{j}$. According to the above transformation, the quantization effects can be transformed into the sector-bounded uncertainties. Defining $\Delta_{k}=$ $\operatorname{diag}\left\{\Delta_{k}^{(1)}, \cdots \Delta_{k}^{(m)}\right\}$, the measurement after quantization is expressed as

$$
z_{k}=\left(I+\Delta_{k}\right) \bar{y}_{k} .
$$

Combining with the measurement description (5), we have

$$
z_{k}=\left(I+\Delta_{k}\right)\left(\Xi_{k} g\left(x_{k}\right)+\xi_{k}\right) .
$$

Letting $\Lambda=\operatorname{diag}\left\{\delta_{1}, \cdots, \delta_{m}\right\}$ and $F_{k}=\Delta_{k} \Lambda^{-1}$, we know that $F_{k}$ is a time-varying matrix satisfying $F_{k} F_{k}^{T} \leq I$.

Remark 1: The recursive filtering scheme subject to degraded measurements and quantization effects is illustrated in

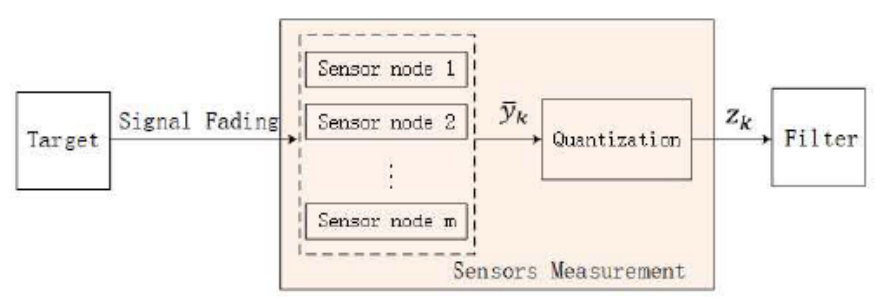

Fig. 2: Filtering for target tracking in wireless localization systems with degraded measurements and quantization effects

Fig. 2. It can be found that the transmissions between the target and the filter could be divided into two stages. The first stage is the sensing process where the position information is measured by sensors suffering from measurement degradations, and the second stage is the communication stage where the measurements would be transmitted to the filter via the communication channel subject to quantization effects.

Remark 2: The signal quantization is a common networkinduced phenomenon, which is mainly caused by the limited transmission capacity in the wireless network. The signal quantization has a significant impact on the system performance [21], [31]. There are two kinds of quantization schemes widely adopted in the literature, namely, the uniform quantization and the logarithmic quantization. Generally speaking, a uniform quantizer has uniformly-spaced quantization levels throughout the range. Hence, small-amplitude signals have the same quantization levels to large-amplitude signals, which implies that small-amplitude signals will lead to a lower signalto-noise ratio (SNR). Compared with the uniform quantizer, logarithmic quantizer could ensure a steady SNR for both small-amplitude signals and large-amplitude signals. In this paper, the logarithmic quantizer is adopted to guarantee a satisfactory SNR for the wireless localization system.

\section{RECURSIVE FILTER DESIGN}

In this section, the recursive filter design is investigated for the time-varying system in the simultaneous presence of degraded measurements and quantization effects. A sufficient condition for the design of filter parameter is established to guarantee an upper bound of the filtering error covariance. Furthermore, such an upper bound can be minimized by properly designing the filter gain parameter.

\section{A. Filter Structure}

In this paper, from (2) and (9), the recursive filter is designed as follows:

$$
\begin{aligned}
& \hat{x}_{k+1 / k}=A_{k} \hat{x}_{k / k} \\
& \hat{x}_{k+1 / k+1} \\
& \quad=\hat{x}_{k+1 / k}+K_{k+1}\left(z_{k+1}-\bar{\Xi}_{k+1} g\left(\hat{x}_{k+1 / k}\right)\right)
\end{aligned}
$$

where $\hat{x}_{k+1 / k}$ and $\hat{x}_{k+1 / k+1}$ are the one-step state prediction and the estimation of $x_{k+1}$ at time $k+1$, respectively. $K_{k+1}$ is the filter gain to be determined. The prediction and filtering 
errors as well as the corresponding covariance matrices are defined by

$$
\left\{\begin{aligned}
\tilde{x}_{k+1 / k} & \triangleq x_{k+1}-\hat{x}_{k+1 / k} \\
\Sigma_{k+1 / k} & \triangleq \mathbb{E}\left\{\tilde{x}_{k+1 / k} \tilde{x}_{k+1 / k}^{T}\right\} \\
\tilde{x}_{k+1 / k+1} & \triangleq x_{k+1}-\hat{x}_{k+1 / k+1}, \\
\Sigma_{k+1 / k+1} & \triangleq \mathbb{E}\left\{\tilde{x}_{k+1 / k+1} \tilde{x}_{k+1 / k+1}^{T}\right\}
\end{aligned}\right.
$$

\section{B. Filter Design}

In the next process, the one-step prediction and filtering error covariances are first calculated, and then an upper bound of the filtering error covariance is derived. In particular, a sequence of positive-definite matrices $P_{k / k}(0 \leq k \leq N)$ is found to ensure $\Sigma_{k / k} \leq P_{k / k}$ and, subsequently, the desired filter gain is obtained by minimizing such an upper bound.

Before proceeding, the following lemmas are recalled which will be used in later developments.

Lemma 1: [13] For any given vectors $X, Y \in \mathbb{R}^{n}$ and a positive scalar $\varepsilon>0$, the following inequality holds

$$
X Y^{T}+Y X^{T} \leq \varepsilon X X^{T}+\varepsilon^{-1} Y Y^{T} .
$$

Lemma 2: [40] For Given matrices $A, H, E$ and $F$ with appropriate dimensions and $F$ satisfying $F F^{T} \leq I$, let $X$ be a symmetric positive definite matrix and $\gamma$ be an arbitrary positive constant such that $\gamma^{-1} I-E X E^{T}>0$. Then, the following inequality holds:

$$
\begin{aligned}
(A & +H F E) X(A+H F E)^{T} \\
& \leq A\left(X^{-1}-\gamma E^{T} E\right)^{-1} A^{T}+\gamma^{-1} H H^{T} .
\end{aligned}
$$

Lemma 3: [11] Let $A$ be a real-valued matrix and $B=$ $\operatorname{diag}\left\{b_{1}, b_{2}, \cdots, b_{p}\right\}$ be a diagonal random matrix. Then, one has

$$
\mathbb{E}\left\{B A B^{T}\right\}=\left[\begin{array}{cccc}
\mathbb{E}\left\{b_{1}^{2}\right\} & \mathbb{E}\left\{b_{1} b_{2}\right\} & \cdots & \mathbb{E}\left\{b_{1} b_{p}\right\} \\
\mathbb{E}\left\{b_{2} b_{1}\right\} & \mathbb{E}\left\{b_{2}^{2}\right\} & \cdots & \mathbb{E}\left\{b_{2} b_{p}\right\} \\
\vdots & \vdots & \vdots & \vdots \\
\mathbb{E}\left\{b_{p} b_{1}\right\} & \mathbb{E}\left\{b_{p} b_{2}\right\} & \cdots & \mathbb{E}\left\{b_{p}^{2}\right\}
\end{array}\right] \circ A
$$

where $\circ$ is the Hadamard product.

Lemma 4: [15] For $0 \leq k \leq N$, suppose that two symmetric matrices $X, Y$ satisfy $0<X \leq Y, S_{k}(X)=$ $S_{k}^{T}(X) \in R^{n \times n}$ and $S_{k}(X) \leq S_{k}(Y)$. Then, the solutions $M_{k}, N_{k}$ to the following difference equations:

$$
M_{k+1} \leq S_{k}\left(M_{k}\right), \quad N_{k+1}=S_{k}\left(N_{k}\right)
$$

with the initial condition $0<N_{0}=M_{0}$ satisfy $M_{k} \leq N_{k}$.

From (2), (10) and (12), the one-step prediction error and the corresponding covariance matrix are obtained as follows:

$$
\begin{aligned}
\tilde{x}_{k+1 / k} & =A_{k} \tilde{x}_{k / k}+B_{k} \omega_{k}, \\
\Sigma_{k+1 / k} & =A_{k} \Sigma_{k / k} A_{k}^{T}+B_{k} R_{\omega} B_{k}^{T} .
\end{aligned}
$$

Moreover, the filtering error is given by:

$$
\begin{aligned}
& \tilde{x}_{k+1 / k+1} \\
& \quad=\tilde{x}_{k+1 / k}-K_{k+1}\left(z_{k+1}-\bar{\Xi}_{k+1} g\left(\hat{x}_{k+1 / k}\right)\right) .
\end{aligned}
$$

Expanding $g\left(x_{k+1}\right)$ in a Taylor series around $\hat{x}_{k+1 / k}$, we derive that

$$
\begin{aligned}
& g\left(x_{k+1}\right) \\
& \quad=g\left(\hat{x}_{k+1 / k}\right)+G_{k+1} \tilde{x}_{k+1 / k}+o\left(\left|\tilde{x}_{k+1 / k}\right|\right),
\end{aligned}
$$

where $G_{k+1} \triangleq \partial g(x) /\left.\partial x\right|_{x=\hat{x}_{k+1 / k}}$ and $o\left(\left|\tilde{x}_{k+1 / k}\right|\right)$ stands for the high-order terms of the Taylor series expansion.

As shown in [16], the high-order terms can be transformed into the following formulation:

$$
o\left(\left|\tilde{x}_{k+1 / k}\right|\right)=C_{k+1} \aleph_{k+1} L_{k+1} \tilde{x}_{k+1 / k},
$$

where $C_{k+1} \in \mathbb{R}^{m \times n}$ is a bounded problem-dependent scaling matrix, $L_{k+1} \in \mathbb{R}^{n \times n}$ is introduced to provide an extra freedom degree to tune the filter, and the unknown time-varying matrix $\aleph_{k+1} \in \mathbb{R}^{n \times n}$ satisfies $\aleph_{k+1} \aleph_{k+1}^{T} \leq I$ accounting for the linearization errors of the measurement model.

Based on (12) and (19)-(21), the filtering error is rearranged as follows:

$$
\begin{aligned}
\tilde{x}_{k+1 / k+1}= & \left(I-K_{k+1} \Psi_{k+1}\right) \tilde{x}_{k+1 / k}-K_{k+1} \\
& \times\left\{\left[\left(I+F_{k+1} \Lambda\right) \Xi_{k+1}-\bar{\Xi}_{k+1}\right] g\left(x_{k+1}\right)\right. \\
& \left.+\left(I+F_{k+1} \Lambda\right) \xi_{k+1}\right\}
\end{aligned}
$$

where

$$
\Psi_{k+1}=\bar{\Xi}_{k+1}\left(G_{k+1}+C_{k+1} \aleph_{k+1} L_{k+1}\right) .
$$

From (12), the covariance of the filtering error is given as follows:

$$
\begin{aligned}
& \Sigma_{k+1 / k+1} \\
&=\left(I-K_{k+1} \Psi_{k+1}\right) \Sigma_{k+1 / k}\left(I-K_{k+1} \Psi_{k+1}\right)^{T} \\
&-\mathbb{E}\left\{( I - K _ { k + 1 } \Psi _ { k + 1 } ) \tilde { x } _ { k + 1 / k } \left[K_{k+1}\right.\right. \\
&\left.\left.\times\left(\left(I+F_{k+1} \Lambda\right) \Xi_{k+1}-\bar{\Xi}_{k+1}\right) g\left(x_{k+1}\right)\right]^{T}\right\} \\
&-K_{k+1} \mathbb{E}\left\{\left[\left(I+F_{k+1} \Lambda\right) \Xi_{k+1}-\bar{\Xi}_{k+1}\right] g\left(x_{k+1}\right)\right. \\
&\left.\times\left[\left(I-K_{k+1} \Psi_{k+1}\right) \tilde{x}_{k+1 / k}\right]^{T}\right\} \\
&+K_{k+1} \mathbb{E}\left\{\left[\left(I+F_{k+1} \Lambda\right) \Xi_{k+1}-\bar{\Xi}_{k+1}\right] g\left(x_{k+1}\right)\right. \\
&\left.\times g^{T}\left(x_{k+1}\right)\left[\left(I+F_{k+1} \Lambda\right) \Xi_{k+1}-\bar{\Xi}_{k+1}\right]^{T}\right\} K_{k+1}^{T} \\
&+K_{k+1}\left(I+F_{k+1} \Lambda\right) R_{\xi}\left(I+F_{k+1} \Lambda\right)^{T} K_{k+1}^{T} .
\end{aligned}
$$

Now, we are in the position to present our main results. The filter parameters are designed such that an optimized upper bound on the filtering error covariance is derived at each sampling instant, and the design procedure is summarized in the following theorem.

Theorem 1: Consider the covariance matrices of the onestep prediction error and the filtering error in (18) and (24). Let $\varepsilon_{1}, \varepsilon_{2}, \varepsilon_{3}, \gamma_{1}, \gamma_{2}$ be positive scalars. Assume that the following two discrete-time Riccati-like difference equations:

$$
\begin{aligned}
& P_{k+1 / k}=A P_{k / k} A^{T}+B_{k} R_{\omega} B_{k}^{T} \\
& P_{k+1 / k+1} \\
& =\left(1+\varepsilon_{1}\right)\left(I-K_{k+1} \bar{\Xi}_{k+1} G_{k+1}\right) \\
& \quad \times\left[P_{k+1 / k}^{-1}-\gamma_{1} L_{k+1}^{T} L_{k+1}\right]^{-1}\left(I-K_{k+1} \bar{\Xi}_{k+1} G_{k+1}\right)^{T} \\
& \quad+K_{k+1}\left\{\left(1+\varepsilon_{1}\right) \gamma_{1}^{-1} \bar{\Xi}_{k+1} C_{k+1} C_{k+1}^{T} \bar{\Xi}_{k+1}^{T}+\left(1+\varepsilon_{1}^{-1}\right)\right. \\
& \quad \times\left[\left(1+\varepsilon_{3}\right) \tilde{\Xi}_{k+1} \circ \Pi_{k+1}+\left(1+\varepsilon_{3}^{-1}\right) \operatorname{tr}\left\{\varphi_{k+1}\right\} I\right] \\
& \left.\quad+\left(R_{\xi}^{-1}-\gamma_{2} \Lambda^{T} \Lambda\right)^{-1}+\gamma_{2}^{-1} I\right\} K_{k+1}^{T}
\end{aligned}
$$


with the initial condition $\Sigma_{0 / 0}=P_{0 / 0}$ have positive definite solutions $\Sigma_{k+1 / k}$ and $\Sigma_{k+1 / k+1}$ such that, for all $0 \leq k \leq N$, the following constraints

$$
\left\{\begin{array}{l}
\gamma_{1}^{-1} I-L_{k+1} P_{k+1 / k} L_{k+1}^{T}>0 \\
\gamma_{2}^{-1} I-\Lambda R_{\xi} \Lambda^{T}>0
\end{array}\right.
$$

are satisfied where

$$
\begin{aligned}
& \tilde{\Xi}_{k+1}=\operatorname{diag}\left\{\nu_{k}^{1}, \nu_{k}^{2}, \cdots, \nu_{k}^{m}\right\}, \\
& \breve{\Xi}_{k+1}=\left[\begin{array}{cccc}
\alpha_{1} & \alpha_{12} & \cdots & \alpha_{1 m} \\
\alpha_{21} & \alpha_{2} & \cdots & \alpha_{2 m} \\
\vdots & \vdots & \vdots & \vdots \\
\alpha_{m 1} & \alpha_{m 2} & \cdots & \alpha_{m}
\end{array}\right] \text {, } \\
& \alpha_{i}=\left(\bar{\beta}_{k}^{i}\right)^{2}+\nu_{k}^{i}, \quad(i=1,2, \cdots, m) \\
& \alpha_{i j}=\bar{\beta}_{k}^{i} \bar{\beta}_{k}^{j}, \quad(i \neq j ; \quad i, j=1,2, \cdots, m) \\
& \varphi_{k+1}=\Lambda\left\{\breve{\Xi}_{k+1} \circ \Pi_{k+1}\right\} \Lambda^{T}, \\
& \Pi_{k+1}=\operatorname{tr}\left[m\left(1+\varepsilon_{2}\right) P_{k+1 / k}\right. \\
& \left.+\sum_{i=1}^{m}\left(1+\varepsilon_{2}^{-1}\right) \breve{x}_{i, k+1} \breve{x}_{i, k+1}^{T}\right] I, \\
& \breve{x}_{i, k+1}=\hat{x}_{k+1 / k}-\bar{x}_{i}^{*} \text {, } \\
& \bar{x}_{i}^{*}=\left[\begin{array}{llll}
x_{1, i}^{*} & 0 & x_{2, i}^{*} & 0
\end{array}\right]^{T} .
\end{aligned}
$$

Then, the desired filter gain $K_{k+1}$ is given by

$$
\begin{aligned}
& K_{k+1} \\
& \quad=\left(1+\varepsilon_{1}\right)\left[P_{k+1 / k}^{-1}-\gamma_{1} L_{k+1}^{T} L_{k+1}\right]^{-1} G_{k+1}^{T} \bar{\Xi}_{k+1}^{T} \\
& \quad \times\left\{\left(1+\varepsilon_{1}\right) \bar{\Xi}_{k+1} G_{k+1}\left[P_{k+1 / k}^{-1}-\gamma_{1} L_{k+1}^{T} L_{k+1}\right]^{-1}\right. \\
& \quad \times G_{k+1}^{T} \bar{\Xi}_{k+1}^{T}+\left(1+\varepsilon_{1}\right) \gamma_{1}^{-1} \bar{\Xi}_{k+1} C_{k+1} C_{k+1}^{T} \bar{\Xi}_{k+1}^{T} \\
& \quad+\left(1+\varepsilon_{1}^{-1}\right)\left[\left(1+\varepsilon_{3}\right) \tilde{\Xi}_{k+1} \circ \Pi_{k+1}\right. \\
& \left.\quad+\left(1+\varepsilon_{3}^{-1}\right) \operatorname{tr}\left\{\varphi_{k+1}\right\} I\right] \\
& \left.\quad+\left(R_{\xi}^{-1}-\gamma_{2} \Lambda^{T} \Lambda\right)^{-1}+\gamma_{2}^{-1} I\right\}^{-1},
\end{aligned}
$$

under which the matrix $P_{k+1 / k+1}$ is an upper bound for $\Sigma_{k+1 / k+1}$, i.e., $\Sigma_{k+1 / k+1} \leq P_{k+1 / k+1}$.

Proof: Based on Lemma 1, the second and third terms of the right-hand side of (24) are rearranged as follows:

$$
\begin{aligned}
\mathbb{E}\{ & -\left[I-K_{k+1} \Psi_{k+1}\right] \tilde{x}_{k+1 / k} \\
& \times\left[K_{k+1}\left[\left(I+F_{k+1} \Lambda\right) \Xi_{k+1}-\bar{\Xi}_{k+1}\right] g\left(x_{k+1 / k}\right)\right]^{T} \\
& -\left[K_{k+1}\left[\left(I+F_{k+1} \Lambda\right) \Xi_{k+1}-\bar{\Xi}_{k+1}\right] g\left(x_{k+1 / k}\right)\right] \\
& \left.\times\left[\left(I-K_{k+1} \Psi_{k+1}\right) \tilde{x}_{k+1 / k}\right]^{T}\right\} \\
\leq & \varepsilon_{1}\left[I-K_{k+1} \Psi_{k+1}\right] \Sigma_{k+1 / k}\left[I-K_{k+1} \Psi_{k+1}\right]^{T} \\
& +\varepsilon_{1}^{-1} K_{k+1} \mathbb{E}\left\{\left[\left(I+F_{k+1} \Lambda\right) \Xi_{k+1}-\Xi_{k+1}\right] g\left(x_{k+1}\right)\right. \\
& \left.\times g^{T}\left(x_{k+1}\right)\left[\left(I+F_{k+1} \Lambda\right) \Xi_{k+1}-\bar{\Xi}_{k+1}\right]^{T}\right\} K_{k+1}^{T} .
\end{aligned}
$$

Then, it follows from (24) and (29) that

$$
\begin{aligned}
& \Sigma_{k+1 / k+1} \\
& \leq\left(1+\varepsilon_{1}\right)\left(I-K_{k+1} \Psi_{k+1}\right) \Sigma_{k+1 / k}\left(I-K_{k+1} \Psi_{k+1}\right)^{T} \\
& \quad+\left(1+\varepsilon_{1}^{-1}\right) K_{k+1} \mathbb{E}\left\{\left[\left(I+F_{k+1} \Lambda\right) \Xi_{k+1}-\bar{\Xi}_{k+1}\right]\right. \\
& \left.\quad \times g\left(x_{k+1}\right) g^{T}\left(x_{k+1}\right)\left[\left(I+F_{k+1} \Lambda\right) \Xi_{k+1}-\bar{\Xi}_{k+1}\right]^{T}\right\}
\end{aligned}
$$

$$
\times K_{k+1}^{T}+K_{k+1}\left(I+F_{k+1} \Lambda\right) R_{\xi}\left(I+F_{k+1} \Lambda\right)^{T} K_{k+1}^{T} .
$$

From Lemma 2 and (27), it is easy to obtain

$$
\begin{aligned}
(I & \left.-K_{k+1} \Psi_{k+1}\right) \Sigma_{k+1 / k}\left(I-K_{k+1} \Psi_{k+1}\right)^{T} \\
= & \left(I-K_{k+1} \bar{\Xi}_{k+1} G_{k+1}-K_{k+1} \bar{\Xi}_{k+1} C_{k+1} \aleph_{k+1} L_{k+1}\right) \\
& \times \Sigma_{k+1 / k}\left(I-K_{k+1} \bar{\Xi}_{k+1} G_{k+1}\right. \\
& \left.-K_{k+1} \bar{\Xi}_{k+1} C_{k+1} \aleph_{k+1} L_{k+1}\right)^{T} \\
\leq & \left(I-K_{k+1} \bar{\Xi}_{k+1} G_{k+1}\right)\left[\Sigma_{k+1 / k}^{-1}-\gamma_{1} L_{k+1}^{T} L_{k+1}\right]^{-1} \\
& \times\left(I-K_{k+1} \bar{\Xi}_{k+1} G_{k+1}\right)^{T} \\
& +\gamma_{1}^{-1} K_{k+1} \bar{\Xi}_{k+1} C_{k+1} C_{k+1}^{T} \bar{\Xi}_{k+1}^{T} K_{k+1}^{T} .
\end{aligned}
$$

Setting $x_{i}^{*}=\left[\begin{array}{ll}x_{1, i}^{*} & x_{2, i}^{*}\end{array}\right]^{T}$ and $\check{x}_{k}=\left[\begin{array}{ll}x_{1}(k) & x_{2}(k)\end{array}\right]^{T}$, one has $g_{i}\left(x_{k}\right)=\left\|\check{x}_{k}-x_{i}^{*}\right\|$, which further results in

$$
\begin{aligned}
\left\|g_{i}\left(x_{k+1}\right)-g_{i}\left(x_{k}\right)\right\| & =\|\| \check{x}_{k+1}-x_{i}^{*}\|-\| \check{\check{x}}_{k}-x_{i}^{*}\|\| \\
& \leq\left\|\check{x}_{k+1}-\check{x}_{k}\right\| \leq\left\|x_{k+1}-x_{k}\right\| .
\end{aligned}
$$

Accordingly, it is easy to check that the nonlinear function $g_{i}\left(x_{k}\right)$ satisfies $g_{i}^{2}\left(x_{k}\right)=\left\|g_{i}\left(x_{k}\right)\right\| \leq\left\|x_{k}-\bar{x}_{i}^{*}\right\|$. Hence, we have

$$
\left\|g\left(x_{k}\right)\right\|=\sqrt{\sum_{i=1}^{m} g_{i}^{2}\left(x_{k}\right)} \leq \sqrt{\sum_{i=1}^{m}\left\|x_{k}-\bar{x}_{i}^{*}\right\|^{2}}
$$

which implies

$$
\begin{aligned}
& \mathbb{E}\left\{g\left(x_{k+1}\right) g^{T}\left(x_{k+1}\right)\right\} \leq \mathbb{E}\left\{\left\|g\left(x_{k+1}\right)\right\|^{2}\right\} I \\
& \leq \mathbb{E}\left\{\sum_{i=1}^{m}\left\|x_{k+1}-\bar{x}_{i}^{*}\right\|^{2}\right\} I \\
& =\sum_{i=1}^{m} \operatorname{tr}\left\{\mathbb{E}\left\{\left(x_{k+1}-\bar{x}_{i}^{*}\right)\left(x_{k+1}-\bar{x}_{i}^{*}\right)^{T}\right\}\right\} .
\end{aligned}
$$

Based on Lemma 1, we arrive at

$$
\begin{aligned}
& \mathbb{E}\left\{\left(x_{k+1}-\bar{x}_{i}^{*}\right)\left(x_{k+1}-\bar{x}_{i}^{*}\right)^{T}\right\} \\
& =\mathbb{E}\left\{\left(\tilde{x}_{k+1 / k}+\breve{x}_{i, k+1}\right)\left(\tilde{x}_{k+1 / k}+\breve{x}_{i, k+1}\right)^{T}\right\} \\
& \leq \mathbb{E}\left\{\left(1+\varepsilon_{2}\right) \widetilde{x}_{k+1 / k} \widetilde{x}_{k+1 / k}^{T}+\left(1+\varepsilon_{2}^{-1}\right) \breve{x}_{i, k+1} \breve{x}_{i, k+1}^{T}\right\} \\
& =\left(1+\varepsilon_{2}\right) \Sigma_{k+1 / k}+\left(1+\varepsilon_{2}^{-1}\right) \breve{x}_{i, k+1} \breve{x}_{i, k+1}^{T},
\end{aligned}
$$

and hence

$$
\mathbb{E}\left\{g\left(x_{k+1}\right) g^{T}\left(x_{k+1}\right)\right\} \leq \Omega_{k+1}
$$

where

$$
\begin{aligned}
\Omega_{k+1}= & \operatorname{tr}\left[m\left(1+\varepsilon_{2}\right) \Sigma_{k+1 / k}\right. \\
& \left.+\sum_{i=1}^{m}\left(1+\varepsilon_{2}^{-1}\right) \breve{x}_{i, k+1} \breve{x}_{i, k+1}^{T}\right] I .
\end{aligned}
$$

According to Lemma 1 and Lemma 3, one has

$$
\begin{aligned}
\mathbb{E}\{ & {\left[\left(I+F_{k+1} \Lambda\right) \Xi_{k+1}-\bar{\Xi}_{k+1}\right] g\left(x_{k+1}\right) g^{T}\left(x_{k+1}\right) } \\
& \left.\times\left[\left(I+F_{k+1} \Lambda\right) \Xi_{k+1}-\bar{\Xi}_{k+1}\right]^{T}\right\} \\
\leq & \mathbb{E}\left\{\left(1+\varepsilon_{3}\right)\left(\Xi_{k+1}-\bar{\Xi}_{k+1}\right) g\left(x_{k+1}\right) g^{T}\left(x_{k+1}\right)\right. \\
& \times\left(\Xi_{k+1}-\bar{\Xi}_{k+1}\right)^{T}+\left(1+\varepsilon_{3}^{-1}\right) F_{k+1} \Lambda \Xi_{k+1} \\
& \left.\times g\left(x_{k+1}\right) g^{T}\left(x_{k+1}\right) \Xi_{k+1}^{T} \Lambda F_{k+1}^{T}\right\}
\end{aligned}
$$




$$
\begin{aligned}
= & \left(1+\varepsilon_{3}\right) \tilde{\Xi}_{k+1} \circ \mathbb{E}\left\{g\left(x_{k+1}\right) g^{T}\left(x_{k+1}\right)\right\}+\left(1+\varepsilon_{3}^{-1}\right) \\
& \times F_{k+1} \Lambda\left(\breve{\Xi}_{k+1} \circ \mathbb{E}\left\{g\left(x_{k+1}\right) g^{T}\left(x_{k+1}\right)\right\}\right) \Lambda F_{k+1} \\
\leq & \left(1+\varepsilon_{3}\right) \tilde{\Xi}_{k+1} \circ \Omega_{k+1}+\left(1+\varepsilon_{3}^{-1}\right) \operatorname{tr}\left\{\phi_{k+1}\right\} I
\end{aligned}
$$

where

$$
\phi_{k+1}=\Lambda\left\{\breve{\Xi}_{k+1} \circ \Omega_{k+1}\right\} \Lambda^{T} .
$$

Furthermore, it follows from Lemma 2 and (27) that

$$
\begin{aligned}
& \left(I+F_{k+1} \Lambda\right) R_{\xi}\left(I+F_{k+1} \Lambda\right)^{T} \\
& \leq\left(R_{\xi}^{-1}-\gamma_{2} \Lambda^{T} \Lambda\right)^{-1}+\gamma_{2}^{-1} I .
\end{aligned}
$$

In view of (30), (31), (37) and (39), we obtain

$$
\begin{aligned}
\Sigma_{k+1 / k+1} & \\
\leq & \left(1+\varepsilon_{1}\right)\left(I-K_{k+1} \bar{\Xi}_{k+1} G_{k+1}\right) \\
& \times\left[\Sigma_{k+1 / k}^{-1}-\gamma_{1} L_{k+1}^{T} L_{k+1}\right]^{-1}\left(I-K_{k+1} \bar{\Xi}_{k+1} G_{k+1}\right)^{T} \\
& +K_{k+1}\left\{\left(1+\varepsilon_{1}\right) \gamma_{1}^{-1} \bar{\Xi}_{k+1} C_{k+1} C_{k+1}^{T} \bar{\Xi}_{k+1}^{T}+\left(1+\varepsilon_{1}^{-1}\right)\right. \\
& \times\left[\left(1+\varepsilon_{3}\right) \tilde{\Xi}_{k+1} \circ \Omega_{k+1}+\left(1+\varepsilon_{3}^{-1}\right) \operatorname{tr}\left\{\phi_{k+1}\right\} I\right] \\
& \left.+\left(R_{\xi}^{-1}-\gamma_{2} \Lambda^{T} \Lambda\right)^{-1}+\gamma_{2}^{-1} I\right\} K_{k+1}^{T} .
\end{aligned}
$$

From (25) and (26), matrix $P_{k+1 / k+1}$ can be viewed as a function of $P_{k / k}$, i.e., $P_{k+1 / k+1}=f_{k}\left(P_{k / k}\right)$, where $f_{k}(\cdot)$ denotes the specific functional relationship between $P_{k+1 / k+1}$ and $P_{k / k}$. Moreover, noting that $\Sigma_{k+1 / k+1} \leq f_{k}\left(\Sigma_{k / k}\right)$, $f_{k}(\cdot)=f_{k}^{T}(\cdot)$ and $P_{0 / 0}=\Sigma_{0 / 0}$, we conclude from Lemma 4 that $\Sigma_{k+1 / k+1} \leq P_{k+1 / k+1}$.

To determine the filter gain, we take the partial derivative of $P_{k+1 / k+1}$ with respect to $K_{k+1}$ and let such a derivative be zero. Then, we have

$$
\begin{aligned}
\partial \operatorname{tr} & \left(P_{k+1 / k+1}\right) / \partial K_{k+1} \\
= & -2\left(1+\varepsilon_{1}\right)\left[P_{k+1 / k}^{-1}-\gamma_{1} L_{k+1}^{T} L_{k+1}\right]^{-1} G_{k+1}^{T} \bar{\Xi}_{k+1}^{T} \\
& +2 K_{k+1}\left\{\left(1+\varepsilon_{1}\right) \Xi_{k+1} G_{k+1}\left[P_{k+1 / k}^{-1}-\gamma_{1} L_{k+1}^{T} L_{k+1}\right]^{-1}\right. \\
& \times G_{k+1}^{T} \bar{\Xi}_{k+1}^{T}+\left(1+\varepsilon_{1}\right) \gamma_{1}^{-1} \bar{\Xi}_{k+1} C_{k+1} C_{k+1}^{T} \bar{\Xi}_{k+1}^{T} \\
& +\left(1+\varepsilon_{1}^{-1}\right)\left[\left(1+\varepsilon_{3}\right) \tilde{\Xi}_{k+1} \circ \Pi_{k+1}+\left(1+\varepsilon_{3}^{-1}\right)\right. \\
& \left.\left.\times \operatorname{tr}\left\{\varphi_{k+1}\right\} I\right]+\left(R_{\xi}^{-1}-\gamma_{2} \Lambda^{T} \Lambda\right)^{-1}+\gamma_{2}^{-1} I\right\}=0 .
\end{aligned}
$$

The filter gain is calculated as shown in (28) which minimizes the upper bound $P_{k+1 / k+1}$ of the filtering error covariance. Therefore, the proof is complete.

Remark 3: In this paper, we focus our attention on the filter design for the nonlinear systems with white Gaussian noise. The recursive filtering is one of the mostly investigated technologies dealing with the state estimation problem with white noise. At each iteration step, we can compute the desired filter parameter based on the solution of two discrete-time Riccati-like difference equations. Note that the solution of the Riccati-like difference equations could be easily obtained by iterative computation, which implies that the feasibility of the filter design could be guaranteed. In Theorem 1, the desired filter parameter is derived based on the value of $P_{k+1 \mid k}$, which could be computed recursively according to two discrete-time Riccati-like difference equations. In the computation process, certain scaling methods have been employed to deal with the inequalities, which would "amplify" the corresponding filtering error covariance. For example, by applying the trigonometric-inequality-based technology, the nonlinear function $g_{i}\left(x_{k}\right)$ satisfies $g_{i}^{2}\left(x_{k}\right)=\left\|g_{i}\left(x_{k}\right)\right\| \leq\left\|x_{k}-\bar{x}_{i}^{*}\right\|$. Even though the computation of the filter parameter leads to the conservatism of the result, it is propitious to realize the filter design conveniently.

Remark 4: It is well known that the traditional Kalman filter has been widely adopted dealing with the estimation problem of the time-varying linear system. The conventional extended Kalman filter has been widely employed to deal with the filtering problem for nonlinear systems, where the involved nonlinearity would be linearized and the corresponding linearization error (i.e. the high-order terms caused by the Taylor expansion) is simply neglected, which inevitably leads to conservatism in certain cases. In [3], Calafiore proposed a more accurate approach to depict the high-order terms in the Taylor series in terms of parameter uncertainties. Similarly, in this paper, we use the deterministic matrix $\aleph_{k}$ and the scaling matrix $L_{k}$ to account for the linearization errors in obtaining the matrix $A_{k}$, which is shown in (21). For more details, we refer the reader to Appendix $\mathrm{C}$ of [3] where a nice interpretation has been given. In addition, It can be seen from (21) that the high-order terms in the Taylor series expansion are commonly bounded, then it is reasonable to regard them as deterministic uncertainties affecting the system matrix $A_{k}$.

\section{Application to the Localization IN THE INDUSTRIAL CITES}

In this section, we present an application example for the wireless localization system to illustrate the effectiveness of the proposed filter design scheme.

Consider the wireless sensors that are deployed in the maintenance region as shown in Fig. 1. The distance measurements are obtained from sensors deployed in the wireless localization system for mine personnel-safety monitor. The "target" is fixed on the safety helmet (the worker must wear the helmet while working in the region), which periodically broadcast its identity and time stamp information through the communication channel. Sensors receive the signal and then generate the measurements which are transmitted to the filter. Based on the received measurements, the estimates are derived via the filtering algorithm and transmitted to the computer for the corresponding cooperative control implementation.

In this experiment, there are 6 sensor nodes performing the target tracking task and the target node is within the radio range of sensors in the maintenance region. As shown in Fig. 1, the coordinates of Sensors 1-6 are $(20,2),(20,12),(10,14)$, $(0,12),(0,2)$ and $(10,0)$, respectively. The target moves along certain elliptical trajectory with a constant speed. The sampling period is $\Delta t_{k}=200 \mathrm{~ms}$. The initial position of the target is $(10,1), \hat{x}_{0 / 0}=\left[\begin{array}{llll}10 & 0.13 & 1 & 0\end{array}\right]^{T}$ and $P_{0 / 0}=I_{4 \times 4}$. For the algorithmic quantizer, we set $\mu_{0}^{(j)}=1$ and $\mathcal{X}_{j}=0.9(j=$ $1, \ldots, 6)$. The statistical characteristics of $\beta_{k}^{i}$ are given as $\bar{\beta}_{k}^{i}=$ 0.8 and $\nu_{k}^{i}=0.04$. Other parameters are chosen as $\gamma_{1}=1$, $\gamma_{2}=100, \varepsilon_{0}=\varepsilon_{1}=\varepsilon_{2}=0.6, C_{k+1}=0.01 I_{6 \times 6}$ and $L_{k+1}=$ $\left[\begin{array}{ll}0.01 I_{4 \times 4} & 0_{4 \times 2}\end{array}\right]^{T}$. Furthermore, the covariance matrices of noises are set as $R_{\omega}=0.01 I_{4 \times 4}$ and $R_{\xi}=0.1 I_{6 \times 6}$.

From Theorem 1, the filter parameters can be designed recursively and the corresponding simulation results are shown in Figs. 3-9. In particular, to reveal the effects of degradation and quantization on the distance measurements, Fig. 3(a) plots 
the measurements of Sensor 3 with and without degradation, and Fig. 3(b) plots the measurements with and without quantization effects. Clearly, the degradation and quantization effects have led to non-negligible errors for the measurement signals.

For purpose of demonstrating the superiority of the proposed filtering algorithm, the mean square error (MSE) is adopted to evaluate the estimation accuracy of the target position. Let $M S E_{i}(k)=(1 / \vartheta) \sum_{j=1}^{\vartheta}\left(x_{i}(k)-\hat{x}_{i}(k)\right)^{2}$ be the mean square error of $x_{i}(k)(i=1,2)$, and $M S E_{3}(k)=$ $(1 / \vartheta) \sum_{j=1}^{\vartheta}\left[\left(x_{1}(k)-\hat{x}_{1}(k)\right)^{2}+\left(x_{2}(k)-\hat{x}_{2}(k)\right)^{2}\right]$ be the mean square error of the target position estimate, where $\vartheta=100$ is the number of Monte Carlo simulations and $\hat{x}_{i}(k)$ is the estimate of $x_{i}(k)$ at time $t_{k}$. Fig. 4 shows the trajectory of $\log \left(M S E_{i}(k)\right)(i=1,2)$ and the corresponding upper bound. It can be seen that the error covariance for each component stays indeed below its upper bound.

Fig. 5 plots the actual moving trajectory and the position estimate of the target in the two-dimensional plane. Let $E_{e}=(1 / N) \sum_{k=1}^{N} \sqrt{\left(x_{1}(k)-\hat{x}_{1}(k)\right)^{2}+\left(x_{2}(k)-\hat{x}_{2}(k)\right)^{2}}$ be the mean square error of the estimation from instant 1 to $N=200$. The mean square error between the actual trajectory and the estimate of the proposed filter is about $E_{e}=0.43 \mathrm{~m}$. Furthermore, the actual coordinates $x_{1}(k)$ and $x_{2}(k)$ of the target position and their estimates are plotted in Fig. 6(a) and Fig. 6(b), respectively, and the maximal errors of the coordinate values and their estimates is about $0.31 \mathrm{~m}$ and $0.24 \mathrm{~m}$, respectively, which reveals the satisfactory performance of the recursive filtering algorithm. Moreover, Fig. 7 shows the filtering performance comparison between the recursive filtering algorithm and the extended Kalman filtering algorithm, and their maximal errors are about $0.58 \mathrm{~m}$ and $0.81 \mathrm{~m}$, respectively. It is obvious that the recursive filtering algorithm has smaller filtering error, which reflects the superiority over the conventional extended Kalman algorithm.

Let us now demonstrate the impact on the filter performance (in terms of the mean square error of the position estimate) from the degraded measurements and the quantization effects, respectively. It can be seen from Fig. 8 that the filtering performance is significantly improved by considering the degraded measurements and quantization effects in the filter design procedure. In addition, the filter performance with different degradation coefficients and quantization densities is depicted in Fig. 9 and Fig. 10, respectively. Fig. 9 plots the trajectory of $\log \left(M S E_{3}(k)\right)$ subject to different degradation coefficients under the same quantization density $\chi_{j}=0.9$, where the best filtering performance is achieved with $\bar{\beta}_{k}^{i}=0.8$ and $\nu_{k}^{i}=0.04$. Fig. 10 shows the trajectory of $\log \left(M S E_{3}(k)\right)$ subject to different quantization density under the same degraded coefficients $\bar{\beta}_{k}^{i}=0.8$ and $\nu_{k}^{i}=0.04$. It can be observed that the filtering performance can be enhanced by adequately accounting for the impacts from measurement degradations and quantization effects on the filter design.

\section{CONCLUSION}

In this paper, in order to enhance the management capability of personnel safety in industrial sites, we have dealt with the wireless target tracking problem for the dynamic personnel localization. In the complicated wireless communication environment, sensor nodes have the limited capability of signal acquisition and transmission, and the signal transmissions are therefore subject to degraded measurements and quantization effects. Our attention has been focused on the recursive filter design issue by taking measurement degradations and quantization effects into special consideration. The proposed filtering algorithm can be implemented recursively, which is suitable for the online computation. Finally, a simulation experiment has been carried out for the wireless personnel tracking system in a mine industry production to demonstrate the effectiveness of the proposed method in practice. Compared with the widely studied quantized $H_{\infty}$ filtering scheme [37], the recursive filtering algorithm developed in this paper could ensure the minimization of the upper bound of the filtering error covariance, thereby leading to the satisfactory local filter performance. Several interesting research topics are to be considered in the future, for example, 1) the filtering problem for uncertain high-order nonlinear systems [46]; 2) the sliding mode filtering problem [14], [35] for wireless localization systems; and 3) the distributed filtering problem for wireless localization systems with stochastic sampling and cyber attacks [7], [28].

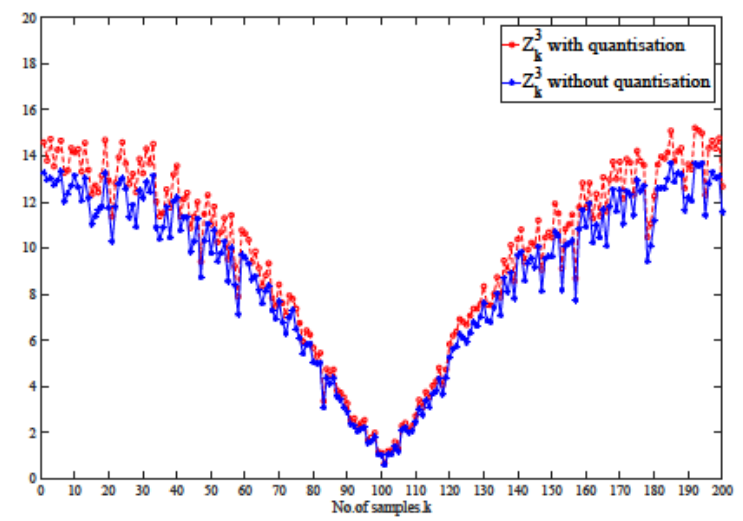

(a) $z_{k}^{3}$ with and without degraded measurement.

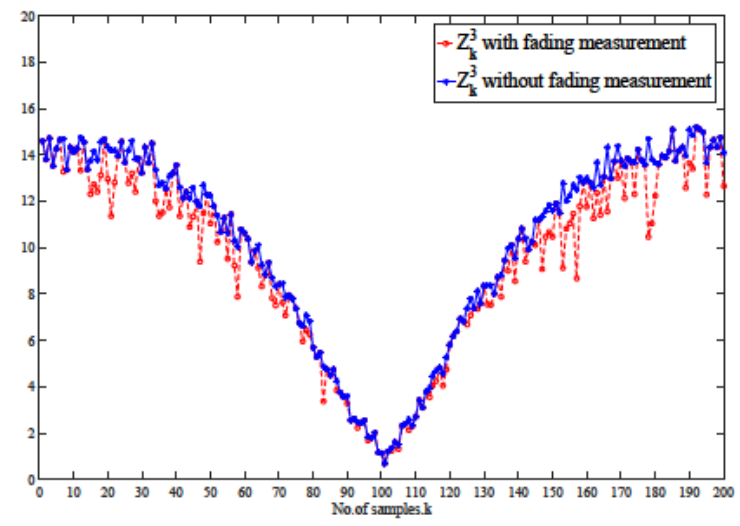

(b) $z_{k}^{3}$ with and without quantization.

Fig. 3: Measurements with and without degraded measurements and quantization effects.

\section{REFERENCES}

[1] A. Abu-Mahfouz, and G. P. Hancke, "Distance bounding: A practical security solution for real-time location systems," IEEE Transactions on Industrial Informatics, vol. 9, no. 1, pp. 16-27, Feb. 2015. 


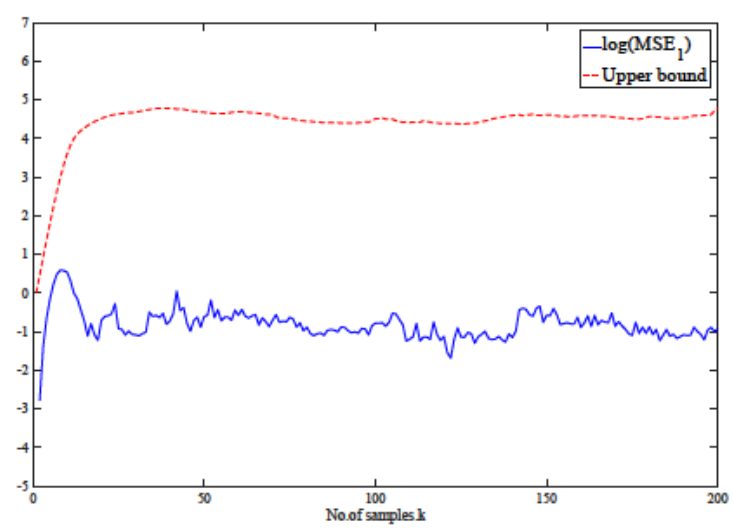

(a) $\log \left(M S E_{1}(k)\right)$ of $x_{1}(k)$ coordinate and its upper bound.

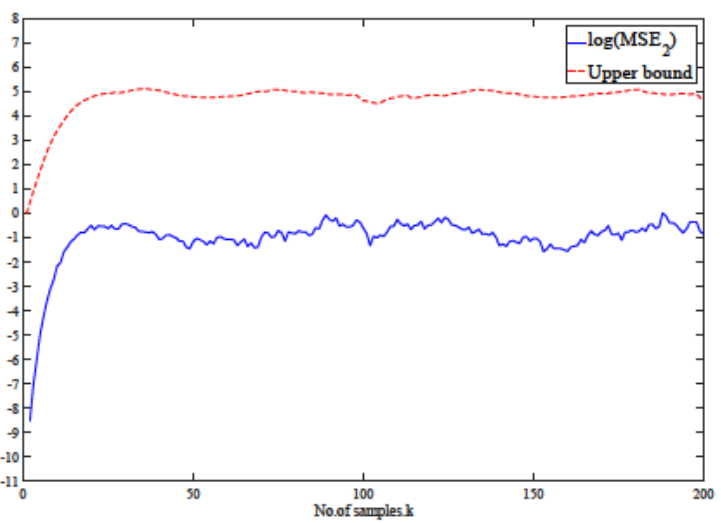

(b) $\log \left(M S E_{2}(k)\right)$ of $x_{2}(k)$ coordinate and its upper bound.

Fig. 4: $\log \left(M S E_{i}(k)\right)(i=1,2)$ and the upper bounds.

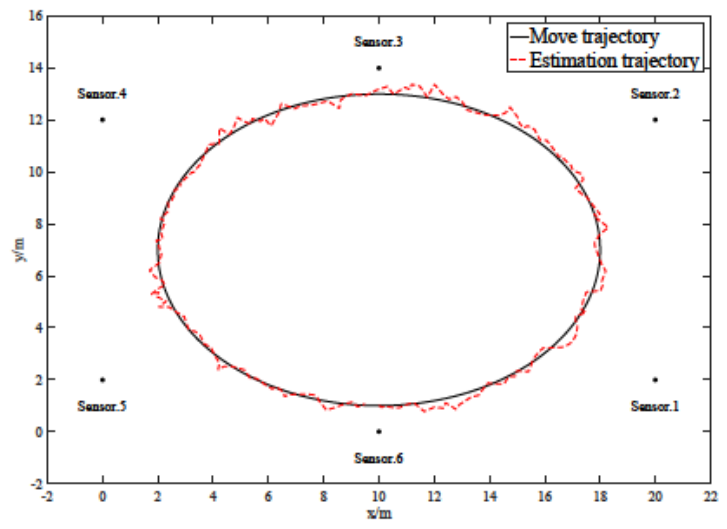

Fig. 5: Actual target trajectory and its estimation.

[2] P. Bahl and V. N. Padmanabhan, "RADAR: an in building RF-based user location and tracking system," in Proceedings of the IEEE International Conference on Computer Communications, pp. 775-784, Tel Aviv, Israel, Mar. 2000.

[3] G. Calafiore, "Reliable Localization Using Set-Valued Nonlinear Filters," IEEE Transactions on Systems, Man, and Cybernetics-Part A Systems and Humans, vol. 35, no. 20, pp. 189-197, Mar. 2005.

[4] Y. Chen, Z. Wang, W. Qian and F. E. Alsaadi, "Asynchronous observerbased $\mathrm{H}$-infinity control for switched stochastic systems with mixed delays under quantization and packet dropouts", Nonlinear Analysis Hybrid Systems, vol. 27, pp. 225-238, Feb. 2018.

[5] L. Cong, and W. Zhuang, "Hybrid TDOA/AOA mobile user location for wideband CDMA cellular systems," IEEE Transactions on Wireless Communications, vol. 1, no. 3, pp. 439-447, Jul. 2002.

[6] K. Derr, and M. Manic, "Wireless sensor networks-node localization for various industry problems," IEEE Transactions on Industrial Informat-

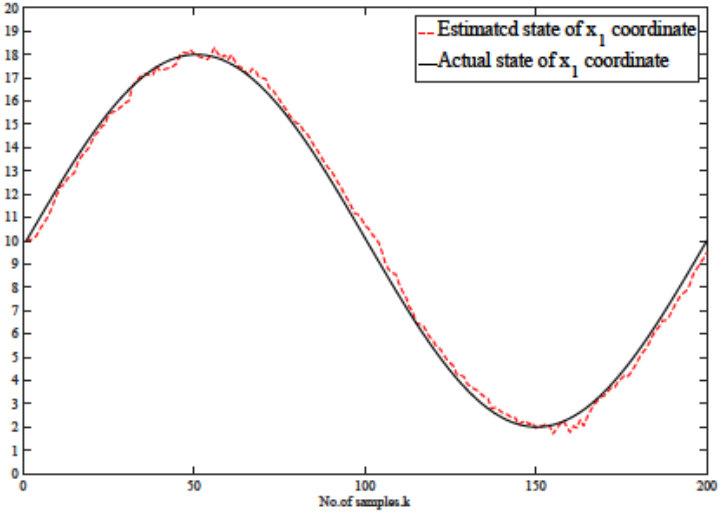

(a) The actual $x_{1}(k)$ coordinate and its estimation.

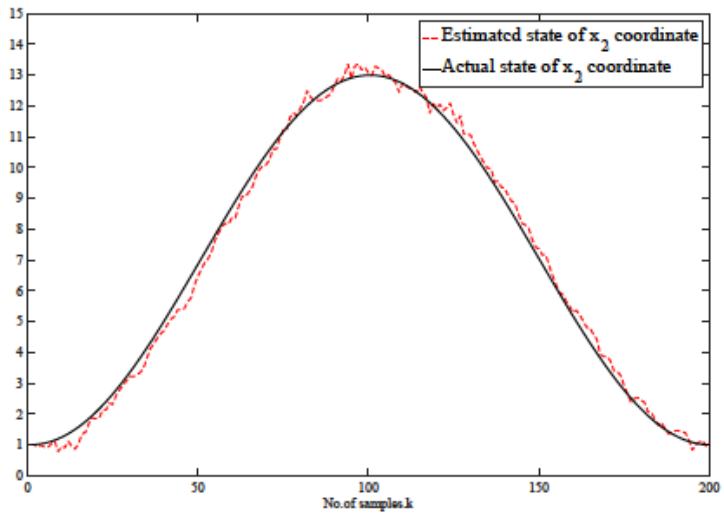

(b) The actual $x_{2}(k)$ coordinate and its estimation.

Fig. 6: The actual state and its estimation.

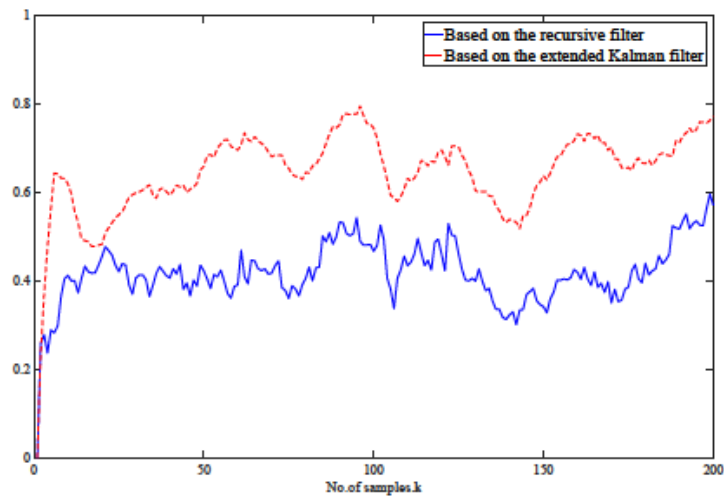

Fig. 7: The comparison of the estimate error of recursive filter and extended Kalman filter.

ics, vol. 11, no. 3, pp. 752-762, Jun. 2015

[7] D. Ding, Z. Wang, D. W. C. Ho and G. Wei, "Distributed recursive filtering for stochastic systems under uniform quantizations and deception attacks through sensor networks", Automatica, vol. 78, pp. 231-240, Apr. 2017.

[8] H. Dong, Z. Wang, S. X. Ding, and H. Gao, "On $H_{\infty}$ estimation of randomly occurring faults for a class of nonlinear time-varing systems with fading channels," IEEE Transactions on Automatic Control, vol. 61, no. 2, pp. $479-484$, Feb. 2016.

[9] H. Dong, Z. Wang, S. X. Ding, and H. Gao, "Event-based $H_{\infty}$ filter design for a class of nonlinear time-varying systems with fading channels and multiplicative noises," IEEE Transactions on Signal Processing, vol. 63 , no. 13, pp. 3387-3395, Jun. 2015.

[10] H. Dong, Z. Wang, and H. Gao, "Distributed filtering for a class of time-varying systems over sensor networks with quantization errors and successive packet dropouts," IEEE Transactions on Signal Processing. 


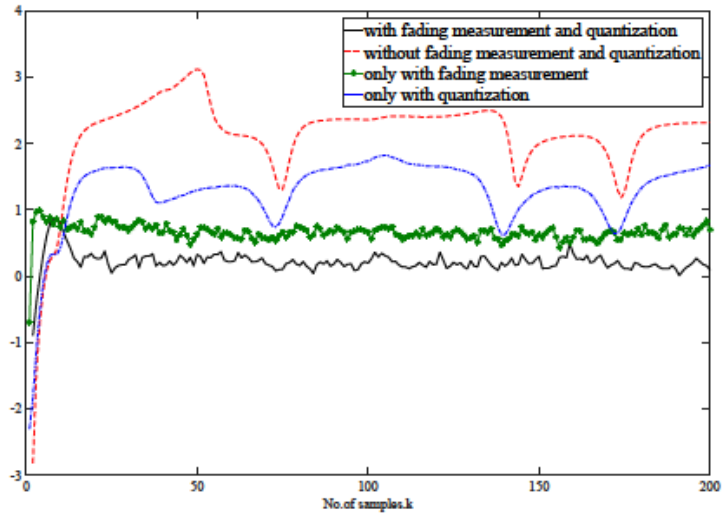

Fig. 8: $\log \left(M S E_{3}(k)\right)$ with and without degraded measurements and quantization effects.

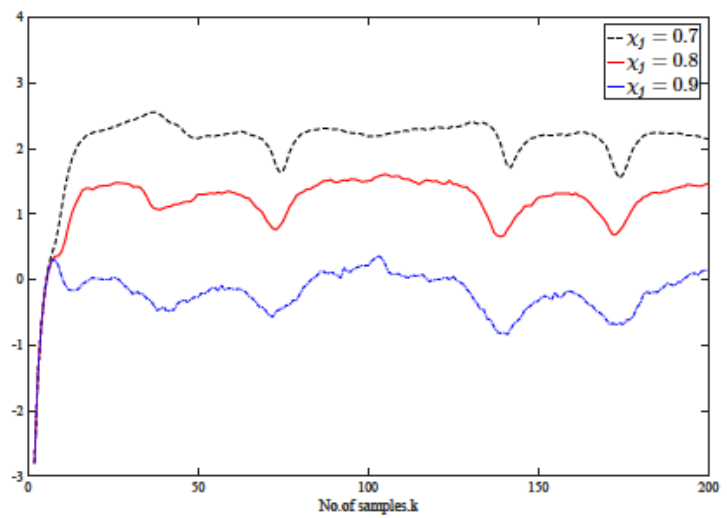

Fig. 9: $\log \left(M S E_{3}(k)\right)$ with different degradation coefficients.

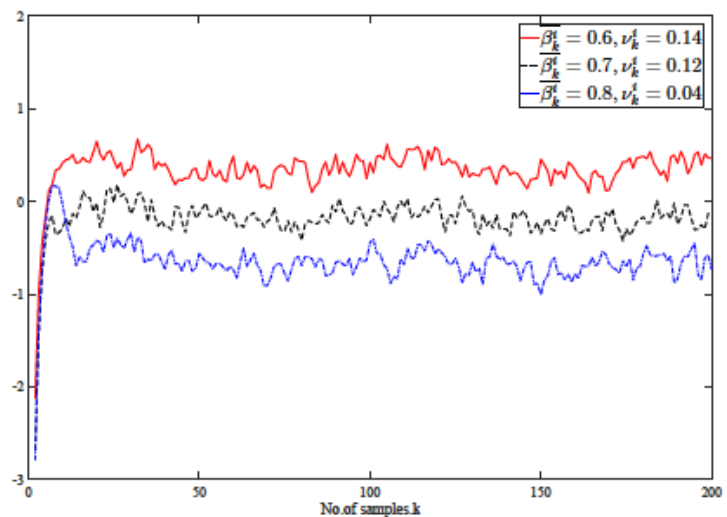

Fig. 10: $\log \left(M S E_{3}(k)\right)$ with different quantization densities.

vol. 60 , no. 6 , pp. $3164-3173$, Jun. 2012 .

[11] R. A. Horn, C. R. Johnson, "Topic in matrix analysis," Cambridge University Press, New York, 1991.

[12] B. Huang, L. Xie, and Z. Yang, "TDOA-based source localization with distance-dependent noises," IEEE Transactions on Wireless Communications, vol. 14, no. 1, pp. 468-480, Jan. 2015.

[13] J. Hu, Z. Wang, H. Gao, and L. K. Stergioulas, "Extended Kalman filtering with stochastic nonlinearities and multiple missing measurements," Automatica, vol. 48, no. 9, pp. 2007-2015, 2012.

[14] J. Hu, Z. Wang, H. Gao and L. K. Stergioulas, "Robust sliding mode control for discrete stochastic systems with mixed time delays, randomly occurring uncertainties, and randomly occurring nonlinearities", IEEE Transactions on Industrial Electronics, vol. 59, no. 7, pp. 3008-3015, Jul. 2012.

[15] L. Hu, Z. Wang, and X. Liu, "Dynamic state estimation of power system with quantization effects: a recursive filter approach," IEEE Transactions on Neural Networks and Learning Systems, vol. 27, no. 8, pp. 16041614, Jul. 2016

[16] X. Kai, C. Wei, and L. Liu, "Robust extended Kalman filtering for nonlinear systems with stochastic uncertainties," IEEE Transactions on Systems Man and Cybernetics-Part A Systems and Humans, vol. 40, no. 2, pp. 399-405, Mar. 2010

[17] L. M. Kaplan, "Global node selection for localization in a distributed sensor network," IEEE Transactions on Aerospace Electronics Systems, vol. 42 , no. 1, pp. 113-135, Jan. 2006

[18] J. Li, H. Dong, F. Han, N. Hou, and X. Li, "Filter design, fault estimation and reliable control for networked time-varying systems: a survey," Systems Science \& Control Engineering, vol. 5, pp. 331-341, 2017.

[19] J. Lin, W. Xiao, F. L. Lewis, and L. Xie, "Energy-efficient distributed adaptive multisensor scheduling for target tracking in wireless sensor networks," IEEE Transactions on Instrumentation and Measurement, vol. 58 , no. 6 , pp. $1886-1896$, Jun. 2009

[20] H. Liu, H. Darabi, P. Banerjee, and J. Liu, "Survey of wireless indoor positioning techniques and systems," IEEE Transactions on Systems, Man, and Cybernetics-Part C Applications and Reviews, vol. 37, no. 6, pp. 1067-1080, Nov. 2007

[21] R. Lu, Y. Xu, A. Xue, and J. Zheng, Networked control with state reset and quantized measurements: observer-based case, IEEE Transactions on Industrial Electronics, vol. 60, no. 11, pp. 5206-5213, Nov. 2013.

[22] Y. Luo, Z. Wang, G. Wei and F. E. Alsaadi, "Robust $H_{\infty}$ filtering for a class of two-dimensional uncertain fuzzy systems with randomly occurring mixed delays", IEEE Transactions on Fuzzy Systems, vol. 25, no. 1, pp. 70-83, Feb. 2017.

[23] L. Ma, Z. Wang, H. K. Lam and N. Kyriakoulis, "Distributed event-based set-membership filtering for a class of nonlinear systems with sensor saturations over sensor networks", IEEE Transactions on Cybernetics, vol. 47 , no. 11, pp. 3772-3783, Nov. 2017

[24] M. McGuire, K. N. Plataniotis, and A. N. Venetsanopoulos, "Data fusion of power and time measurements for mobile terminal location," IEEE Transactions on Mobile Computing, vol. 4, no. 2, pp. 142-153, 2005.

[25] J. M. Pak, C. K. Ahn, Y. S. Shmaliy, and M. T. Lim, "Improving reliability of Particle filter-based localization in wireless sensor networks via hybrid Particle/FIR filtering," IEEE Transactions on Industrial Informatics, vol. 11, no. 5, pp. 1089-1098, Oct. 2015.

[26] N. Patwari, J. N. Ash, S. Kyperountas, A. O. Hero, R. L. Moses, and N. S. Correal, "Locating the nodes: cooperative localization in wireless sensor networks," IEEE Signal Processing Magazine, vol. 22, no. 4, pp. 54-69, Apr. 2005.

[27] N. Patwari, A. O. Hero, M. Perkins, N. S. Correal, and R. J. O'Dea, "Relative location estimation in wireless sensor networks," IEEE Transactions on Signal Processing, vol. 51, no. 8, pp. 2137-2148, Aug. 2003.

[28] C. Peng, J. Zhang, and H. Yan, "Adaptive event-triggering $H_{\infty}$ load frequency control for network-based power systems," IEEE Transactions on Industrial Electronics, vol.65, no.2, pp.1685-1694, Feb. 2018.

[29] C. Peng and Q.-L. Han, "On designing a novel self-triggered sampling scheme for networked control systems with data losses and communication delays", IEEE Transactions on Industrial Electronics, vol. 63, no. 2, pp. 1239-1248, Feb. 2016

[30] Y. B. Shalom, X. R. Li, and T. Kirubarajan,"Estimation with application to tracking and navigation," Wiley, vol. 53, no. 6, pp. 993-999, 2001.

[31] L. Sheng, Z. Wang, W. Wang and F. E. Alsaadi, "Output-feedback control for nonlinear stochastic systems with successive packet dropouts and uniform quantization effects", IEEE Transactions on Systems, Man, and Cybernetics - Systems, vol. 47, no. 7, pp. 1181-1191, Jul. 2017.

[32] N. J. Thomas, D. G. M. Cruickshank, and D. I Laurenson, "Performance of a TDOA-AOA hybrid mobile location system," in Proceedings of the 2th International Conference on $3 G$ Mobile Communication Technologies, pp. 216-220, London, England, Mar. 2001.

[33] X. Wang, M. Fu, and H. Zhang, "Target tracking in wireless sensor networks based on the combination of KF and MLE using distance measurements," IEEE Transactions on Mobile Computing, vol. 11, no. 4, pp. 567-576, Apr. 2012.

[34] X. Wang, H. Zhang, and M. Fu, "Collaborative target tracking in WSNs using the combination of maximum likelihood estimation and Kalman filtering," Journal of Control Theory and Applications, vol. 11, no. 1, pp. 27-34, 2013.

[35] Y. Wang, H. Shen, H. R. Karimi, and D. Duan, "Dissipativitybased fuzzy integral sliding mode control of continuous-time TS fuzzy systems," IEEE Transactions on Fuzzy Systems, in press, DOI:10.1109/TFUZZ.2017.2710952.

[36] Z. Wang, H. Dong, B. Shen, and H. Gao, "Finite-horizon $H_{\infty}$ filtering with missing measurements and quantization effects," IEEE Transactions on Automatic Control, vol. 58, no. 7, pp. 1707-1718, Jul. 2013.

[37] Y. Wei, J. Qiu and H. R. Karimi, Quantized $H_{\infty}$ filtering for continuous-time Markovian jump systems with deficient mode infor- 
mation, Asian Journal of Control, vol.17, no.5, pp.1914-1923, Sep. 2015.

[38] A. J. Weiss, "Direct geolocation of wideband emitters based on delay and Doppler," IEEE Transactions on Signal Processing, vol. 59, no. 6, pp. 2513-2521, Jun. 2011.

[39] W. Xiao, L. Xie, J. Chen, and L. Shue, "Multi-step adaptive sensor scheduling for target tracking in wireless sensor networks," in Proceedings of the IEEE International Conference on Acoustics Speech and Signal Processing, pp. 705-708, Toulouse, France, May 2006.

[40] L. Xie, Y. C. Soh, and C. E. de Souza, "Robust Kalman filtering for uncertain discrete-time systems," IEEE Transactions on Automatic Control, vol. 39, no. 6, pp. 1310-1314, Jun. 1994.

[41] Y. Xu, Z. Wang, D. Yao, R. Lu, and C. Su, "State estimation for periodic neural networks with uncertain weight matrices and Markovian jump channel states," IEEE Transactions on Systems, Man, and Cybernetics Systems, in press, DOI: 10.1109/TSMC.2017.2708700.

[42] H. Yan, F. Qian, F. Yang, and G. Guo, " $H_{\infty}$ fault detection for networked mechanical spring-mass systems with incomplete information," IEEE Transactions on Industrial Electronics, vol.63, no.9, pp.56225631, Sep. 2016.

[43] F. Yin, C. Fritsche, F. Gustafsson, and A. M. Zoubir, "TOA-based robust wireless geolocation and Cramér-Rao lower bound analysis in harsh LOS/NLOS environments," IEEE Transactions on Signal Processing, vol. 61, no. 9, pp. 2243-2255, May 2013.

[44] Y. Yuan, H. Yuan, Z. Wang, L. Guo, and H. Yang, "Optimal control for networked control systems with disturbances: a delta operator approach", IET Control Theory \& Applications, vol. 11, no. 9, pp. 1325-1332, 2017.

[45] D. Zhang, L. Yu, and W. Zhang, "Energy efficient distributed filtering for a class of nonlinear systems in sensor networks," IEEE Sensors Journal, vol. 15, no. 5, pp. 3026-3036, May 2015.

[46] X. Zhao, P. Shi, X. Zheng, and J. Zhang, "Intelligent tracking control for a class of uncertain high-order nonlinear systems," IEEE Transactions on Neural Networks and Learning Systems, vol. 27, no. 9, pp. 1976-1982, Sep. 2016.

[47] L. Zou, Z. Wang, Q.-L. Han and D. H. Zhou, "Ultimate boundedness control for networked systems with Try-Once-Discard protocol and uniform quantization effects", IEEE Transactions on Automatic Control, vol. 62 , no. 12 , pp. 6582-6588, Dec. 2017. 